\title{
Terahertz Waveform Synthesis via Optical Pulse Shaping
}

\author{
Yongqian Liu, Sang-Gyu Park, and A. M. Weiner
}

\begin{abstract}
We describe the principles of free-space terahertz waveform synthesis by using a programmable optical pulse shaper to drive a photoconducting dipole antenna. We illustrate this technique using several experimental examples, including manipulation of the amplitude and the phase of ultrafast terahertz waveforms as well as generation of ultrafast bit sequences at terahertz frequencies. We present a theory which accurately predicts the shapes of the terahertz waveforms produced in our experiments. In addition to the controllability of terahertz radiation, we have shown that optical pulse shaping can be used to avoid saturation of the terahertz field at highpeak power and increase generation efficiencies for terahertz radiations at selected, narrow-band frequencies.
\end{abstract}

\section{INTRODUCTION}

I $\mathrm{N}$ RECENT YEARS, there has been a growing interest in generating terahertz $(\mathrm{THz})$ radiation for both scientific and technological applications [1]-[3]. The coherent terahertz radiation field provides a unique tool to access the millimeter to submillimeter spectral region, which is quite difficult to achieve with conventional microwave and Far-IR techniques [4]-[6]. This has opened up new methods for material characterizations based on coherent time-domain spectroscopy [5], [6], high-speed circuit testing [7], and imaging and ranging of objects using submillimeter-wave radiation [8], [9]. Various techniques have been been developed and demonstrated to launch and detect such terahertz radiation, including dipole and large aperture photoconductive antennas, optical rectification using unbiased semiconductors and electrooptic crystals, and photoexcitation of coherent charge oscillations in semiconductor heterostructures [1]-[16]. Over the years, researchers have concentrated on improving the speed and power of terahertz radiation from both the generation and detection points of view. Detected terahertz pulses can be as fast as a single-cycle or even half-cycle and can span a spectral range up to near Mid-IR [5], [17]-[19]. The peak power of the terahertz radiation can reach a few hundred Watt using optimum design of terahertz transmitters in combination with high power amplified laser pulses [12], [18], [20]. Terahertz radiation is usually generated from a single short optical pulse, resulting in a short burst of terahertz radiation and a broad-band spectrum. More recently, narrow-band terahertz radiation has also been generated using interference of chirped

Manuscript received September 18, 1996; revised December 5, 1996. This work was supported by National Science Foundation under Grant 9404677 PHY and by the U.S. Air Force Office of Scientific Research under Contract AFOSR F49620-95-1-0490.

The authors are with the School of Electrical and Computer Engineering, Purdue University, West Lafayette, IN 47907-1285 USA.

Publisher Item Identifier S 1077-260X(96)09587-1. optical pulses [21]. To extend the flexibility of terahertz radiation for some other applications, such as coherent control of molecular states in the terahertz-frequency range and submillimeter communications, it is also desirable to have capabilities of generating arbitrarily shaped millimeter and submillimeter waveforms. Some attempts have been made to generate simple terahertz waveforms through coherent control of charge oscillations in quantum-well structures and through spatially patterning the photocurrent distributions in large aperture photoconducting antennas [22]-[26]. In the former case, the ability to craft terahertz waveforms is limited by the built-in charge oscillations. In the latter case, an angular slice of the whole radiated power from the large aperture antenna was detected resulting in loss of signal strengths. For arbitrary shaping of terahertz waveforms, photoconductive approaches are more applicable. Recent developments in femtosecond pulse-shaping technology, which provides the ability to synthesize ultrashort optical waveforms according to specification [27]-[33], have significant implications for optoelectronic devices such as terahertz radiation systems. Since the terahertz radiation depends on the exciting optical intensity profiles, femtosecond pulse-shaping technology could make it possible to engineer the shape of terahertz waveforms. Similar to applications of optical pulse shaping for ultrafast optical communications [31] and coherent control [35], the ability to craft terahertz radiation fields has great potential for coherent control of dynamical processes such as molecular bond-breaking through interaction with rotational or vibrational motions [35], [36] and submillimeter-wave interconnect systems based on terahertz radiation.

In this paper, we describe a technique for terahertz waveform synthesis based on radiation from ultrafast current surges in photoconductive switches excited by shaped optical pulses. Our technique provides a simple way to generate flexible terahertz waveforms and detect the radiation beam with minimum loss of powers. For completeness, we will sketch a theory that calculates the antenna response to a single optical pulse using parameters related to the photoconductive materials. We will derive the theory that quantitatively predicts terahertz radiation waveforms from the antenna response measured in response to a single optical pulse. Our theory shows for the first time to our knowledge that complicated terahertz waveforms can be calculated based on the shape of the input optical pulses and the measured single-pulse terahertz response. We then demonstrate several examples of terahertz waveform synthesis, illustrating control of both the phase and the amplitude of generated terahertz signals, and compare the experimental 
results with calculations using our theory. We obtain excellent agreement between theory and experiment without any external adjustable parameters. We also show that optical pulse sequences can be used to generate tunable, narrowband terahertz radiation. Aside from the utility of crafting terahertz waveforms to our specifications, we demonstrate that the power spectral density at the peak of the narrow-band terahertz spectrum can be significantly enhanced by using multiple pulse sequences instead of single optical pulses [37]. These results can be attributed to the avoidance of saturation effects, which can occur for example when the radiated field amplitude is sufficient to partially screen the applied bias field.

This paper is organized as follows. We describe in Section II a generalized theory which relates the shape of the terahertz radiation field from a photoconductive antenna to the exciting optical intensity waveforms. Section III describes the experimental apparatus for optical pulse shaping and for generation of shaped terahertz radiation fields. In Section IV, we present several examples of shaped terahertz radiation waveforms that are generated using optical pulse sequences produced using a programmable liquid crystal modulator inside an optical pulse shaper. Section V presents generation of tunable narrowband terahertz radiation generated using microlithographicallyfabricated phase masks inside a pulse shaper. Section VI presents enhancement of the narrow-band terahertz radiation spectral amplitude by this technique. Finally, Section VII summarizes the experimental results.

\section{THEORY}

The principle of terahertz waveform engineering in our work is to control the radiation field by changing the optical pulse shape. The desired optical waveforms are generated by a femtosecond pulse shaper and used to excite a photoconducting dipole antenna. The pulse shaper consists of a grating and lens pair with a spatially patterned mask placed in the Fourier plane of the pulse shaper to tailor the optical frequency spectrum. The pulse shape at the output of the apparatus is determined by the Fourier transform of the pattern transferred by the masks onto the spectrum. We use $e_{\text {in }}(t)$ and $e_{\text {out }}(t)$ to denote the electric field profiles of the pulses before and after the pulse shaper, respectively. The pulse emerging from the pulse-shaping apparatus is given by [31]

$$
\int d t e_{\text {out }}(t) e^{-i \omega t}=E_{\text {out }}(\omega)=E_{\text {in }}(\omega) H(\omega)
$$

where $E_{\text {out }}(\omega)$ and $E_{\text {in }}(\omega)$ are the Fourier transforms of $e_{\text {out }}(t)$ and $e_{\mathbf{m}}(t)$, respectively, and $H(\omega)$ is the effective frequency filter implemented by the masks within the pulse shaper setup. The output profile of the shaped optical pulses is written as $I_{\text {out }}(t) \sim\left|e_{\text {out }}(t)\right|^{2}$, with Fourier transform [32]

$$
\int d t I_{\text {out }}(t) e^{-i \omega t}=\tilde{I}_{\text {out }}(\omega)=\int d \Omega E_{\text {out }}^{*}(\Omega) E_{\text {out }}(\Omega+\omega) \text {. }
$$

Here, $\tilde{I}_{\text {out }}(\omega)$ denotes the Fourier transform of $I_{\text {out }}(t)$; it does not denote the magnitude squared of the Fourier transform of the field.

The generated terahertz radiation originates from the transient current induced by the optical pulses. In the linear response regime, the radiation field is given by the convolution of the exciting optical pulse intensity and the terahertz system response. The response function of a free-space terahertz system consists of the transmitter response, the terahertz-wave propagation function $P(\omega)$ which accounts for the frequency and geometry dependent coupling between transmitter and receiver, and the receiver response. Now let us consider a pair of antennas consisting of a transmitter and a receiver. Similar to the transmitter response function previously discussed by [4], [38], the transient current density in the transmitter can be described by

$$
\begin{aligned}
j_{T}(t) & =\sigma_{s}(t) E_{\mathrm{dc}} \sim I_{T}(t) \otimes R_{T}(t) \\
& =\int_{0}^{t} d t^{\prime} I_{T}\left(t^{\prime}\right)\left(1-e^{-\left(t-t^{\prime}\right) / \tau_{c}}\right) e^{-\left(t-t^{\prime}\right) / \tau_{T}}
\end{aligned}
$$

where $\sigma_{s}(t)$ is the surface conductivity, $E_{\mathrm{dc}}$ is the bias field, $\otimes$ denotes convolution, and $I_{T}(t)$ is the exciting optical pulse intensity on the transmitter. $R_{T}(t)$ is the photocurrent response function of the transmitter, which we take to have the following form

$$
R_{T}(t) \sim u(t)\left(1-e^{-\left(t-t^{\prime}\right) / \tau_{c}}\right) e^{-\left(t-t^{\prime}\right) / \tau_{T}} .
$$

Here, $u(t)$ is a unit step function, $\tau_{T}$ is the carrier lifetime of the photoconductive material used to fabricate the transmitter, and $\tau_{c}$ is the "collision time" appearing in Drude theory of photoexcited carriers with the lattice. Similar to [4], $R_{T}(t)$ represents a finite rise time for the current during the initial ballistic acceleration of the photoexcited carriers, followed by exponential decay due to carrier trapping or recombination. Equation (3) is valid assuming a constant bias field $E_{\mathrm{dc}}$ and in the absence of any saturation mechanisms. The spectral amplitude of the transient current is given by

$$
J_{T}(\omega)=\tilde{I}_{T}(\omega) \tilde{R}_{T}(\omega)
$$

where $\tilde{R}_{T}(\omega)$ is the Fourier transform of the transmitter photocurrent response $R_{T}(t)$. In general, the radiation field can be related to the transient current pulse by a characteristic frequency-dependent antenna response $Z_{T}(\omega)$

$$
E_{\mathrm{rad}}(\omega)=Z_{T}(\omega) \tilde{I}_{T}(\omega) \tilde{R}_{T}(\omega)
$$

In the case of a dipole antenna where the dimension of the dipole is much smaller than the average radiation wavelength, $Z_{T}(\omega) \sim i \omega$ since the radiation field follows the time-derivative of the current pulse.

The radiated field at the receiver applies a transient bias across the receiver photoconductive gap, which is sampled by illuminating with an optical probe pulse. The detected signal is the time-integrated photocurrent, i.e., total charge $Q(\tau)$, resulting from this sampling operation, as a function of the delay $\tau$ of the probe pulse with respect to the incoming terahertz field. The time-dependent receiver photocurrent may be written as the product of the induced bias voltage across the photoconductive gap $v_{r}(t)$ with the time-dependent conductance $g(t-\tau)$ induced by the delayed probe pulse arriving at time $\tau$

$$
j_{r}(t)=v_{r}(t) g(t-\tau)
$$


The induced bias voltage is related to the incoming terahertz field by

$$
V_{r}(\omega)=Z_{R}(\omega) E_{\mathrm{rad}}(\omega) P(\omega)
$$

where $V_{r}(\omega)$ is the Fourier transform of $v_{r}(t)$ and $Z_{R}(\omega)$ is the frequency-dependent receiver antenna response function (again $Z_{R}(\omega) \sim i \omega$ for a short dipole). The probe-induced conductance $g(t)$ in response to a probe pulse at $\tau=0$ is given by

$$
g(t) \sim \int_{-\infty}^{t} I_{R}\left(t^{\prime}\right) R_{R}\left(t-t^{\prime}\right) d t^{\prime} .
$$

Here $I_{R}(t)$ is the temporal profile of the probe pulse (in our experiment the probe pulse is the same as the input pulse) and $R_{R}(t)$ is the photoconductance impulse response function of the receiving antenna, given by

$$
R_{R}(t) \sim u(t) e^{-t / \tau_{R}}
$$

where $\tau_{R}$ is the carrier lifetime of the receiver. For the receiver we neglect the finite rise time for the photocurrent. This assumption allows us to simplify the rather complicated expression which would be required to describe the transient photocurrent in the case of finite photocurrent rise time with both the bias field and the carrier density time-dependent. This assumption should be justified at least in part since the integration over the transient photocurrent, yielding $Q(\tau)$, will be dominated by the width of the photocurrent response, which is closely related to the carrier lifetime.

The expression for $Q(\tau)$ can now be written as follows:

$$
Q(\tau)=\int d t v_{r}(t) g(t-\tau)
$$

The Fourier transform of $Q(\tau)$, denoted $\tilde{Q}(\omega)$, assumes a particularly convenient form

$$
\begin{aligned}
\tilde{Q}(\omega) & =\int d \tau Q(\tau) e^{-i \omega \tau}=V_{R}(\omega) G^{*}(\omega) \\
& \sim Z_{T}(\omega) Z_{R}(\omega) P(\omega) \tilde{R}_{T}(\omega) \tilde{R}_{R}^{*}(\omega) \tilde{I}_{T}(\omega) \tilde{I}_{R}^{*}(\omega) .
\end{aligned}
$$

Here, $\tilde{R}_{R}(\omega)$ and $\tilde{I}_{R}(\omega)$ are the Fourier transform of $R_{R}(t)$ and $I_{R}(t)$, respectively. For our studies, we used shaped optical pulses to excite the transmitter and unshaped optical pulses to gate the receiver. In this case, from (1) and (2),

$$
\begin{aligned}
& \tilde{I}_{T}(\omega)=\int d \Omega E_{\text {in }}^{*}(\Omega) E_{\text {in }}(\Omega+\omega) H^{*}(\Omega) H(\Omega+\omega) \\
& \tilde{I}_{R}(\omega)=\tilde{I}_{\text {in }}(\omega)=\int d \Omega E_{\text {in }}^{*}(\Omega) E_{\text {in }}(\Omega+\omega)
\end{aligned}
$$

where $\tilde{I}_{\mathrm{in}}(\omega)$ is the Fourier transform of the intensity profile of the input unshaped optical pulse. We can now rewrite the expression for $\widetilde{Q}(\omega)$ as

$$
\tilde{Q}(\omega)=S(\omega) H^{\prime}(\omega)
$$

where $S(\omega)$ is the characteristic terahertz system response resulting from identical unshaped pump and probe optical pulses $I_{\mathrm{in}}(t)$ and is given by

$$
S(\omega) \sim Z_{T}(\omega) Z_{R}(\omega) P(\omega) \tilde{R}_{T}(\omega) \tilde{R}_{R}^{*}(\omega)\left|\tilde{I}_{\text {in }}(\omega)\right|^{2} .
$$

$H^{\prime}(\omega)$ is the effective response function of the pulse shaper in terms of the optical intensities and is defined by

$$
H^{\prime}(\omega)=\frac{\tilde{I}_{T}(\omega)}{\tilde{I}_{\mathrm{in}}(\omega)} .
$$

Equations (15)-(17) represent one of our key results. They indicate that the measured signal $Q(\tau)=\frac{1}{2 \pi} \int d \omega \tilde{Q}(\omega) e^{i \omega t}$ is equal to the convolution of the terahertz response function observed for unshaped pulses with an effective "intensity impulse response" function for the pulse shapers. If the unshaped input pulses are very short compared to the photocurrent response functions, the terahertz response function $S(\omega)$ is approximately independent of the input pulse duration; in this case the form $Q(\tau)$ observed in response to a shaped pump pulse is just the unshaped pulse terahertz response convolved directly with the shaped intensity profile.

From the above equations, it is obvious that linear filtering of the optical field can be used to modify terahertz radiation field. Thus, the ability to shape optical pulses can be extended to control over the shape of terahertz waveforms, provided that the response of the terahertz system is sufficiently fast. We notice that although the terahertz response is linear in the shaped intensity $I_{T}(t)$ and the effective intensity filter function $H^{\prime}(\omega)$, it is nonlinear in the shaped optical field and in the actual filtering function $H(\omega)$. Therefore, in order to find pulse-shaping filter $H(\omega)$ which will produce a specified terahertz waveform, one must invert the nonlinear equations given by (13)-(17). These are limitations to the range of terahertz waveforms which can be synthesized. For example, since the terahertz-generation process is driven by the optical intensity, which is always nonnegative, it is not possible to invert the sign of the terahertz waveform through optical pulse shaping (assuming fixed transmitter bias field $E_{\mathrm{dc}}$ ). This problem can be resolved, however, if coherence is incorporated into the emitter part (e.g., generation through difference frequency mixing). It is also not possible to generate terahertz waveforms with significantly greater spectral content than generated in unshaped pulse experiments. We also note that for terahertz waveforms, which can be synthesized, in general more than one pulse-shaping filter $H(\omega)$ can be used to produce the required shaped pulse $I_{T}(t)$, since the phase profile of the optical field is a free parameter. This has the practical implication that $H(\omega)$ can often be implemented as a phase-only filter for high optical energy efficiency [34].

In order to compare our experiments with theory, we use (15) to calculate the expected terahertz waveforms with a given mask function in the pulse shaper, using either of two approaches. The first approach is based on first principle calculations to directly calculate both the shaped optical pulse from the intended mask filter function using (13) and the terahertz system response $S(\omega)$ from the materials parameters using (16).

As an example, consider the terahertz system response with both pump and probe unshaped, Fig. 1(a) shows a typical measurement of the unshaped pulse terahertz response. A typical intensity cross correlation measurement of the pump and probe pulses is shown in the inset. Fig. 1(b) shows the calculated terahertz response using (16). $P(\omega)=1$ is assumed 

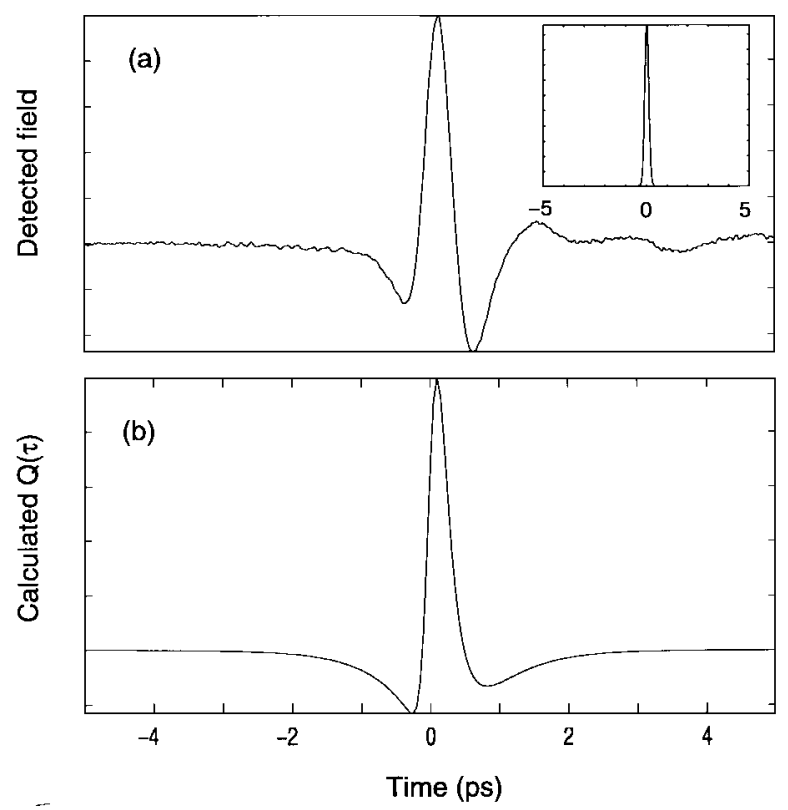

Fig. 1. (a) Typical characteristic terahertz system response with single-pulse optial excitation. The inset shows the cross correlation of unshaped pump pulses with pulses direcly from the laser as the reference. (b) Calculated characteristic terahertz system response from (16) using $\tau_{T}=0.6 \mathrm{ps}, \tau_{R}=$ $0.6 \mathrm{ps}$, and $\tau_{c}=0.3 \mathrm{ps}$ with a 150 -fs input-optical pulse.

here for ease of calculation. The agreement between data and calculation is reasonably good with the following assumed values for the material parameters: $\tau_{T}=0.6 \mathrm{ps}, \tau_{R}=0.6$ $\mathrm{ps}$, and $\tau_{c}=0.3 \mathrm{ps}$ (note that these values are not uniquely determined). The main difference between these two plots is the more pronounced oscillatory features evident in Fig. 1(a), which is most likely due to frequency-dependent propagation effects of the terahertz beam, which is neglected in this calculation.

This approach can be extended to the case of shaped pulses by computing $H^{\prime}(\omega)$ from (17) and the design value of the masking function $H(\omega)$.

The second approach, which is the one we follow in the rest of the paper, uses the measured form of $H^{\prime}(\omega)$ together with the measured terahertz system response $S(\omega)$ obtained from single optical pulse experiments. An expression for $H^{\prime}(\omega)$ can be obtained experimentally using the intensity cross correlation data. Expressions for the cross correlations of the shaped and the unshaped pulses $I_{\text {in }}(t)$ with unshaped pulses $I_{\text {in }}(t)$ directly from the laser are given respectively as follows:

$$
\begin{aligned}
& X_{T}(\tau)=\int d t I_{T}(t) I_{\mathrm{in}}(t-\tau) \\
& X_{\mathrm{in}}(\tau)=\int d t I_{\mathrm{in}}(t) I_{\mathrm{in}}(t-\tau)
\end{aligned}
$$

The Fourier transforms of the intensity cross correlations are given by

$$
\begin{aligned}
& \tilde{X}_{T}(\omega)=\int d \tau X_{T}(\tau) e^{-i \omega \tau}=\tilde{I}_{T}(\omega) \tilde{I}_{\mathrm{in}}^{*}(\omega) \\
& \tilde{X}_{\mathrm{in}}(\omega)=\int d \tau X_{\mathrm{in}}(\tau) e^{-i \omega \tau}=\tilde{I}_{\mathrm{in}}(\omega) \tilde{I}_{\mathrm{in}}^{*}(\omega) .
\end{aligned}
$$

The $H^{\prime}(\omega)$ is determined by dividing the Fourier transformed cross correlation data

$$
H^{\prime}(\omega)=\frac{\tilde{I}_{T}(\omega)}{\tilde{I}_{\mathrm{in}}(\omega)}=\frac{\tilde{X}_{T}(\omega)}{\tilde{X}_{\mathrm{in}}(\omega)}
$$

This second approach using only measured data takes into account any nonideal mask responses from pulse shaper as well as lack of complete knowledge about the photoconducting material parameters. The examples shown later in this paper verify that this calculational approach produces an excellent agreement with shaped pulse experiments, with no adjustable parameters.

Note that we could also shape the optical pulse incident on the receiver instead of on the transmitter. Although this would not generate a shaped terahertz beam, it will produce a shaped experimental measurement $Q(\tau)$, with the shape related to a time-reversed version of the optical probing pulse. Using both shaped pump and probe pulses will produce a $Q(\tau)$ waveform related to the intensity cross-correlation function between $I_{T}(t)$ and $I_{R}(t)$. Note also that our calculation should not be limited to terahertz waveforms design using pulse shapers but could also be applied to general cases of terahertz waveform enginnering based on optical pulse profiles generated using other techniques.

\section{EXPERIMENTAL APPARATUS}

Our experiments are based on the enabling technologies of femtosecond pulse shaping [27]-[29], [31] and LT-GaAs based photoconducting dipole antennas [39]. We used a Ti-sapphire oscillator (Spectra Physics Tsunami) producing 100-fs pulses at a repetition rate of $80 \mathrm{MHz}$ around a center wavelength of $800 \mathrm{~nm}$. A schematic diagram of the experimental arrangement is shown in Fig. 2. The femtosecond laser output is split into two beams. One of them acts as the pump to a transmitting antenna while the other is the probe beam gating a receiving antenna. A pulse shaper is used in the pump beam to design the exciting optical pulse intensity profiles. The pulse-shaping apparatus, similar to [27]-[28], consists of a pair of 1800line/mm gratings placed at the focal planes of a unit magnification achromatic confocal lens (15-cm focal lengths) pair. The optical pulse design is accomplished by either a programmable liquid crystal modulator (LCM) manufactured by Cambridge Research Inc. or a fixed microlithographically-fabricated phase mask similar to [32]. The LCM we used is a combination of two 128-element liquid crystal modulators that can filter both the amplitude and the phase of the optical spectrum [29]. The LCM was controlled through a homemade computer graphical interface to set the driving voltage of each modulator element through use of a predetermined calibration table relating the filter function $H(\omega)$ and the driving voltage. Intensity profiles of the shaped pulses are measured by cross correlation via second harmonic generation, using pulses directly from the laser as the reference. The shaped optical pulses are focused to a $\sim 10-\mu \mathrm{m}$ spot on the transmitting antenna. The dipole antenna structure consists of a pair of Ti-Au transmission lines (separated by $30 \mu \mathrm{m}$ ) with a small gap in the middle $(5 \mu \mathrm{m})$. The dipole antennas are fabricated on LT-GaAs 


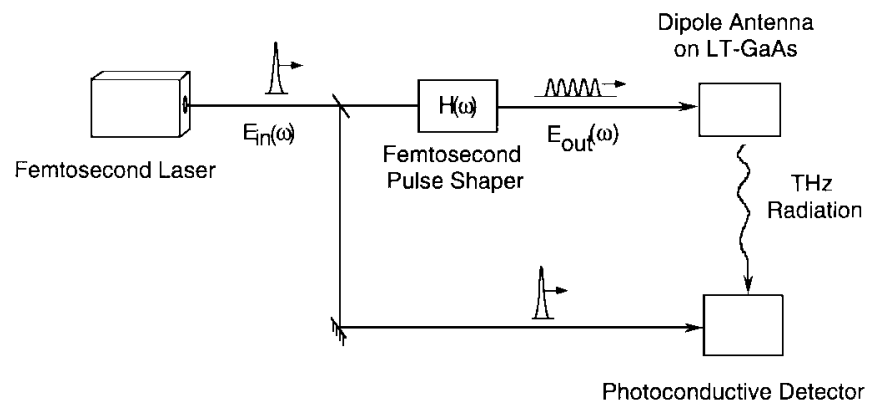

Fig. 2. Schematic diagram of experimental arrangement.

with subpicosecond carrier lifetimes and are mounted on high-resistivity silicon hyperhemispherical lens with a $10-\mathrm{mm}$ diameter and a center thickness of $6.4 \mathrm{~mm}$. In our experiments, the transmitting antenna is biased by a dc power supply with $\sim 25 \mathrm{~V}$. The terahertz radiation propagates in free space and is focused by another silicon hyperhemispherical lens onto a similar photoconducting dipole antenna which acted as the receiver. The receiver was placed $\sim 10 \mathrm{~cm}$ away from the transmitter to detect the far-field radiation. The unshaped optical probe beam was used as a trigger to sample the terahertz radiation field sensed by the detector. The current signal, proportional to the radiated field, was amplified by a current preamplifier and recorded by a lock-in amplifier. The terahertz radiation field was mapped out as a function of time by varying the delay between the excitation pulse and the sampling pulse.

\section{TERAHERTZ WAVEFORM SYNTHESIS}

In this section, we demonstrate several examples of terahertz waveform synthesis by using different optical pulse sequences. We will show that both the phase and amplitude profiles of terahertz waveforms can be modulated. In order to compare our experimental results with calculations, for every data set we measured the characteristic terahertz system response $S(\omega)$ using single-pulse excitation in order to account for any changes due to day-to-day variations in the system alignment. In each case, we also performed intensity cross correlation measurements of both the shaped and unshaped optical pulses using pulses directly from the laser as the reference. A typical pump average power of $\sim 7 \mathrm{~mW}$ and probe power of $\sim 20 \mathrm{~mW}$ were used in the following experiments.

\section{A. Terahertz Internal Phase Modulation}

As a first example, we demonstrate control over the phases of a six-cycle terahertz radiation waveform. We accomplish this by designing a sequence of six optical pulses with different separations. The filter function we used is the Fourier transform of a sequence of delta functions separated by a certain time spacing $\tau$. For instance, a six-pulse sequence with equal time-spacing between each pulse is given by

$$
H(\omega)=e^{-2 i \omega \tau}+e^{-i \omega \tau}+1+e^{i \omega \tau}+e^{2 i \omega \tau}+e^{3 i \omega \tau}
$$

where $\omega$ corresponds to the optical frequency. Controlling the internal phase of the resultant terahertz waveform can be
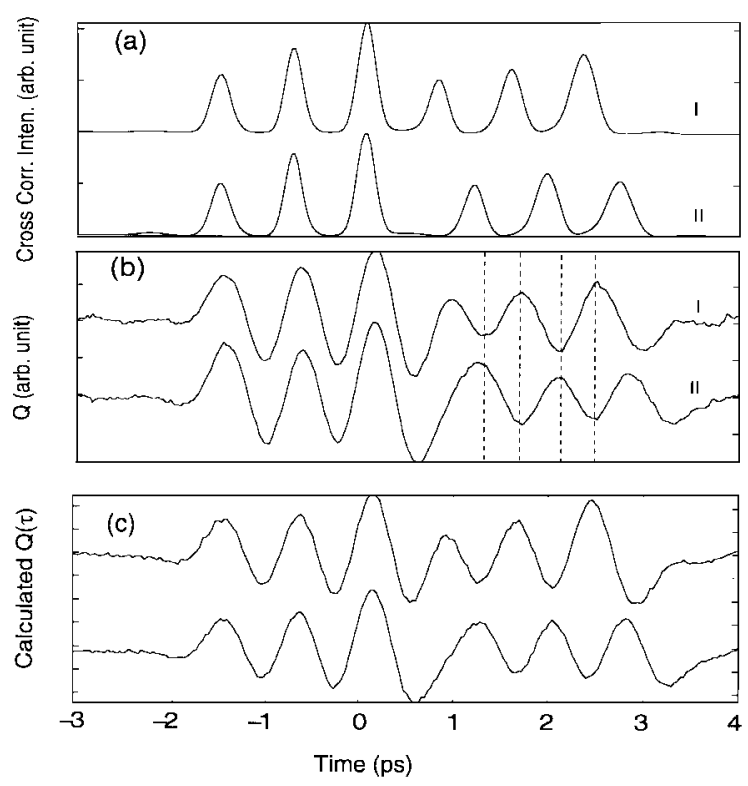

Fig. 3. (a) Measured cross correlation of two designed six-pulse sequences: (I) equally time-spaced (II) an extra time delay at the fourth pulse. (b) Measured terahertz radiation from the two pulse sequences. (c) Calculated terahertz radiation based on (15) and (17) using measured characteristic terahertz system response and cross-correlations data.

achieved simply by modifying the separations between pulses. As long as the terahertz response function is fast enough to respond to each pulse, the phases of individual terahertz cycles are changed as a result of the optical pulse timing.

Fig. 3(a) shows cross correlations of two six-pulse optical pulse sequences from the pulse shaper. The first (I) has equal pulse spacing (period $\tau \sim 0.8 \mathrm{ps}$ ); The second (II) has an extra time delay at the fourth pulse ( $1.5 \tau$ instead of $\tau$ ). The terahertz waveforms resulting from these excitation pulse sequences are shown in Fig. 3(b). The first pulse sequence generated a quasi-sinusoidal terahertz waveform with a constant frequency corresponding to the repetition rate of the six-pulse sequence. The second waveform generated a similar terahertz waveform but with a $\pi$ phase shift occurring between the third and fourth terahertz cycle. The phase reversals at the instants of time shown by the dashed lines are clearly visible. Internal phase modulation, as demonstrated in this simple example, could potentially be used to generate orthogonal terahertz waveforms with reduced cross-talk for communication applications.

Fig. 3(c) shows the calculated radiation field based on (15) and (17) using the measured terahertz system response from an unshaped pump pulse as well as the cross correlation measurements of the unshaped pulse and the shaped optical pulse sequences [see Fig. 3(a)]. An excellent agreement with the experimentally observed terahertz waveforms is observed. We emphasize that there are no adjustable parameters in these calculations; only the measured single-pulse terahertz response and the measured intensity cross correlations of unshaped and shaped optical pulses are needed. The agreement between data and calculations without adjustable parameters validates the role of the optical pulse shaper in terahertz waveform synthesis as described in Section II. We have also performed first principles calculations of the terahertz 
waveform using assumed exponential carrier lifetime for the terahertz transmitting and receiving antennas, and using the intended optical filter function [e.g., (23)]. Although these first principles calculations show qualitative agreement with the data, there are also discrepances. These discrepancies can arise principally from two sources. First, the amplitude of the optical pulses in the actual pulse sequences [Fig. 3(a)] are not equal, which is not expected from (23); and second, the terahertz system response resulting from single-pulse excitation is not precisely reproduced by the model as discussed in Section II. The use of measured cross correlations and the measured terahertz system response removes these uncertainties and produces closer agreement with the experiments.

\section{B. Terahertz Amplitude Modulation}

In this section, we consider two examples of terahertz amplitude modulation which show the versatility of terahertz waveform engineering based on pulse-shaping techniques.

Time-Division Multiplexing: One application of our technique is to generate terahertz waveforms forming binary bit sequences, which could be used for example for high speed chip-to-chip or board-to-board interconnect systems involving high-speed time-division multiplexed (TDM) data. Compared to previous work by [22], which first demonstrated such a possibility using space-to-time mapping based on antenna arrays, our optical pulse-shaping approach can be extended to more complicated encoding schemes. Fig. (4) shows one example of our data, where we used two different femtosecond pulse sequences to generate terahertz waveforms encoded as two different 4-bit words. The optical pulse sequences consist of up to four pulses with pulse separation of $\sim 2$ ps as shown by the cross correlation trace in Fig. 4(a). Respectively, they represent 1111 and 1101 4-bit sequences with each peak of the pulses representing one. These modulated signals at optical frequencies $(\sim 800 \mathrm{~nm})$ can be directly transferred to submillimeter waveforms as shown by the corresponding terahertz measurement in Fig. 4(b). Note that in the terahertz experiments, it is the electric field that is detected, in contrast to the optical measurements in which intensity is detected.

Fig. 4(c) shows the calculated radiation field based on (15) and (17) using measured terahertz system response from an unshaped pump pulse and the cross correlation measurements of the shaped optical pulse sequences as shown in Fig. 4(a). Again, we can see almost a perfect match between the calculation and the experimentally observed results shown in Fig. 4(b). From these calculations, we expect that the maximum speed of the terahertz modulation is determined by the slower of the data rate generated by the pulse shaper and the response time of the terahertz antenna system.

Narrow-Band Terahertz Amplitude Modulation: The previous section demonstrated binary amplitude modulation of a terahertz pulse sequence. In fact, it is also easy to implement variable (gray-level) amplitude modulation of the terahertz waveform through grey-level control of the optical intensity. To demonstrate this, we create a narrow-band terahertz waveform by using an optical spectral phase filter as described below. The filter consists of periodic repetitions of
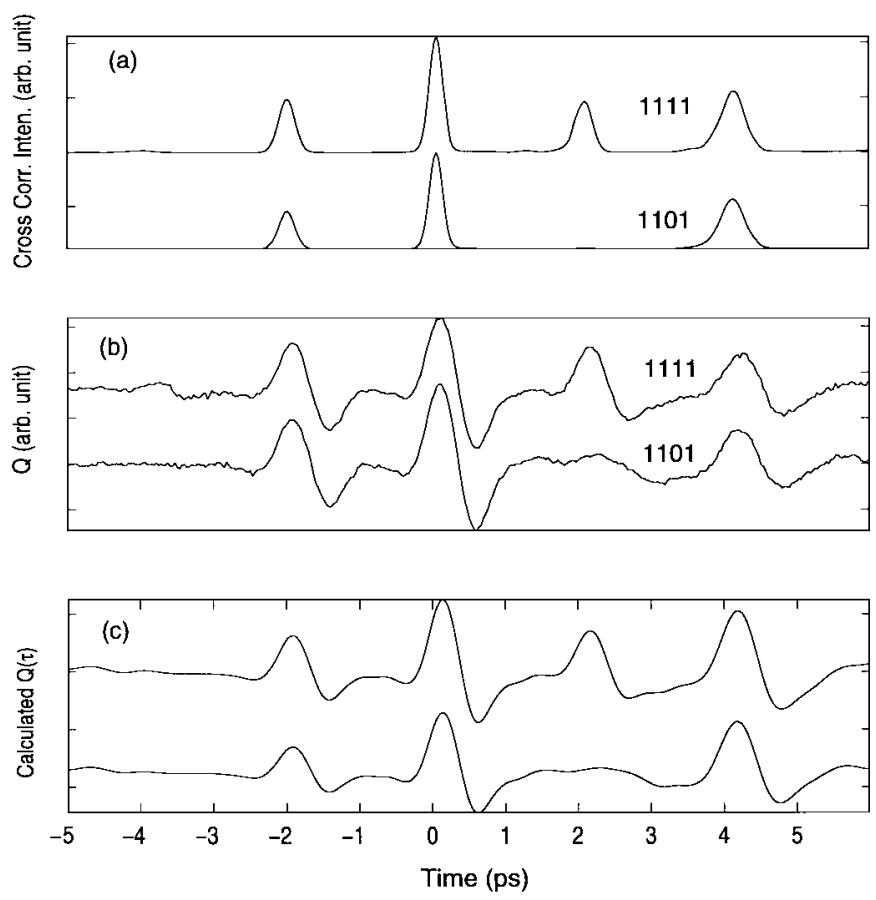

Fig. 4. Terahertz time-domain multiplexing of two 4-bit words. (a) Measured cross corelation of input optical pulses. (b) Measured terahertz radiation as a result of the two encoded sequences. (c) Calculated terahertz radiation of the two 4-bit words based on terahertz system response using measured characteristic terahertz system response and cross correlations data.

a 15-element $M$-sequence phase function generated using the programmable liquid crystal modulator. The $M$-sequence is given by $\{000100110101111\}$ where the 0 's and 1's represent phases of zero and $\Delta \Phi$ respectively. This pattern is repeated periodically to determine all 128 elements of the LCM. As has been previously demonstrated [27], [28], [32], such phase functions produce narrow-band optical pulse sequences with the amplitude of the center pulse controlled by varying the phase shift $\Delta \Phi$. Fig. 5(a) shows the cross correlation of a series of narrow-band optical pulse sequences. The different phase shifts $\Delta \Phi=\pi, 1.20 \pi, 1.31 \pi$, and $1.43 \pi$ result in a dramatic change in the peak amplitude of the center pulse which varies from close to zero to a dominant high amplitude. Shown in Fig. 5(b) are the corresponding terahertz waveform data. As expected, the amplitude of the center-cycle terahertz radiation is modulated as a result of the optical pulse sequence design, varying from a near zero to a large peak. Similar to the previous cases, Fig. 5(c) shows the calculated radiation fields based on (15) and (17). The excellent agreement between the calculations and the experiments once again supports our theoretical model. The design of narrow-band terahertz radiation with variable amplitude modulation could have applications in coherent molecular control to selectively generate rotational modes [35], [36].

\section{TUNABle NARROW-BAND TERAHERTZ RADIATION}

The experiments on narrow-band terahertz radiation demonstrated above can be also expanded to add tunability. This can be accomplished simply by designing similar phase masks with different spatial periodicities. The relation of the optical 


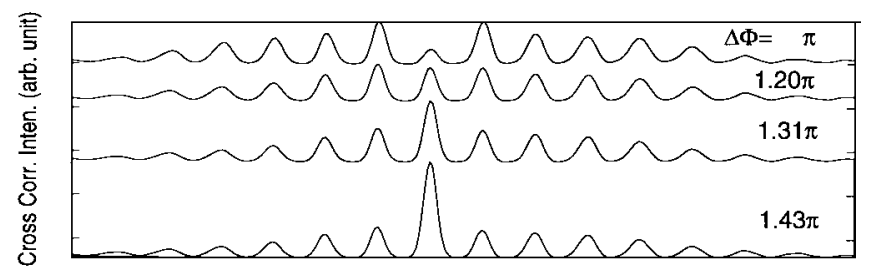

(a)

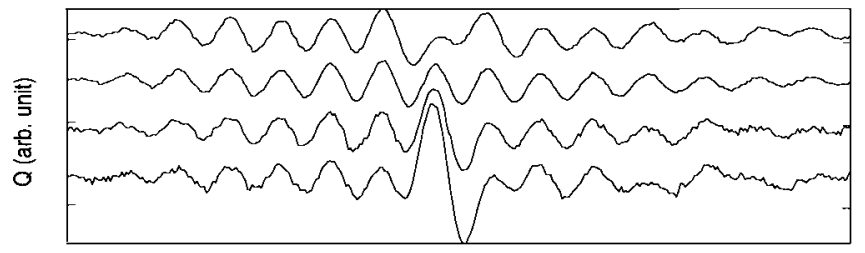

(b)

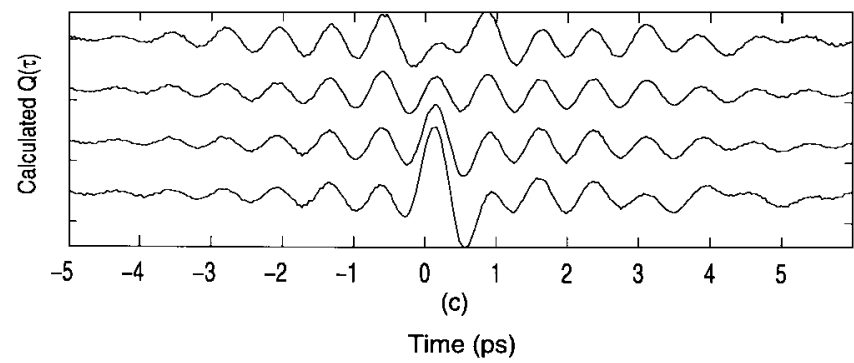

Fig. 5. Narrow-band amplitude modulation of terahertz waveforms. (a) Measured cross correlation of input optical pulses with variable change in phase $(\Delta \Phi)$ of a $M$-sequence filter function. (b) Measured amplitude modulated terahertz waveform corresponding to the above input pulses. (c) Calculated terahertz radiation based on terahertz system response and cross-correlation data.

repetition rate and phase mask periodicity can be described by

$$
\nu_{0}=\frac{c d \cos \left(\theta_{d}\right) \Delta x}{F \lambda^{2}}
$$

where $\nu_{0}$ is the repetition frequency of a designed pulse sequence, $c$ is the speed of light, $d$ is the grating period, $\theta_{d}$ is the diffraction angle off the first grating of pulse shaper, $F$ is the focal length of lens after the grating, $\lambda$ is the optical wavelength, and $\Delta x$ is the spatial period of the $M$-sequence phase mask.

In order to demonstrate generation of tunable narrow-band radiation, we tuned the center frequency $\nu_{0}$ through control of the mask spatial period $\Delta x$. This was accomplished using a fixed, microlithographically fabricated $M$-sequence phase mask similar to [27], [32], [33]. The mask contains a series of approximately 20 one-dimensional phase patterns, where each individual pattern is designed as a periodic repetition of an $M$ sequence, as described above. The spatial periodicity varies from pattern to pattern, and therefore, the pulse repetition period $\nu_{0}$ can be tuned by physically translating the mask to place different patterns in front of the spatially dispersed optical beam. For comparison, the mask also contains a blank, constant phase area which produces a single unshaped optical pulse. The experiment shown below used a pump power of $\sim 14 \mathrm{~mW}$. One interesting and important feature of such a phase mask is illustrated in Fig. 6, which shows the Fourier transforms of intensity cross correlation measurements of an unshaped pulse and of a shaped pulse sequence. The key

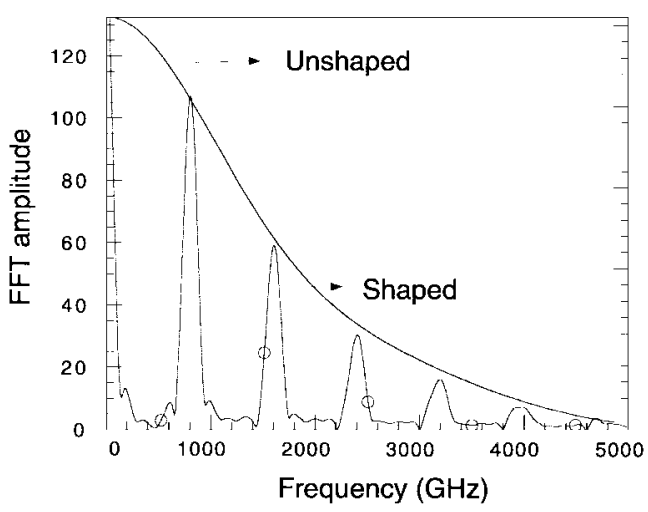

Fig. 6. Fourier transform of measured intensity cross correlation of single and multiple optical pulses.

point is that the periodic optical phase mask acts as a periodic narrow-band filter which selects only certain frequency components from $I(\omega)$, the Fourier transform of the intensity (not from the Fourier transform of the field). The amplitude of the selected frequencies are the same as for the unshaped pulse. This can be understood with reference to (1) and (2), which show that $I(\omega)$ is given by the correlation function of the masked optical spectrum $E(\omega)$. As long as the filter $H(\omega)$ is a periodic phase-only function, the correlation is unchanged by the phase mask for frequencies $\nu_{n}=n \nu_{0}$, where $n$ is an integer and $\nu_{0}$ is determined by the spatial periodicity of the mask through (24). For frequencies $\nu_{n} \neq n \nu_{0}$, the correlation is low (this is guaranteed by the properties of $M$-sequences), and therefore $I(\omega)$ is low also.

Fig. 7 shows the terahertz radiation measured (solid lines) using this phase mask for several representative cases: $\Delta x=$ (a) $0.875 \mathrm{~mm}$, (b) $0.717 \mathrm{~mm}$, (c) $0.625 \mathrm{~mm}$. The terahertz radiation shows quasi-sinusoidal waveforms with center frequencies equal to the repetition frequencies of the optical pulse trains generated from each phase pattern. The phase shift is $\Delta \Phi=0.84 \pi$ in order to provide a central pulse amplitude consistent with a smooth pulse sequence envelope. The center frequency of the narrow-band terahertz radiation is tuned as a result of varying the spatial period of the mask patterns, which changes the optical pulse repetition frequency. Dashed lines in Fig. 7 represent the respective terahertz waveforms from calculations based on (15) and (17) using the measured terahertz system response [Fig. 7(a)] together with measured cross correlations (not shown). The calculations show a smaller temporal window due to shorter temporal scans in cross correlation measurements. Once again, we obtain a very good agreement between theory and experiment, including the shapes of the individual terahertz features making up the quasi-sinusoidal burst.

Fig. 8 shows the Fourier transforms of the time-domain terahertz waveforms from the previous figure. As expected, the single-pulse radiation is broad-band with spectral content extending up to $2.5 \mathrm{THz}$ (limited by our detector bandwidth). The narrow-band radiation from the pulse sequences shows both a first-harmonic and second-harmonic peak within our bandwidth. It can be noticed that the spectral peak amplitude at the first harmonic of the narrow-band radiation is enhanced 


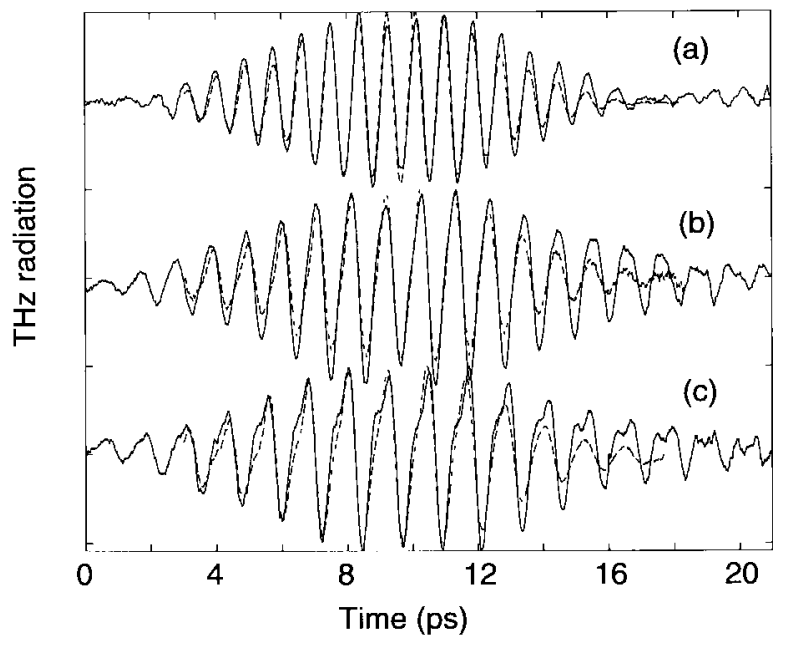

Fig. 7. Measured (solid lines) and calculated (dashed lines) terahertz radiation with fixed periodic $M$-sequence phase mask: (a) $\Delta x=0.875 \mathrm{~mm}$, (b) $\Delta x=0.717 \mathrm{~mm}$, (c) $\Delta x=0.625 \mathrm{~mm}$.

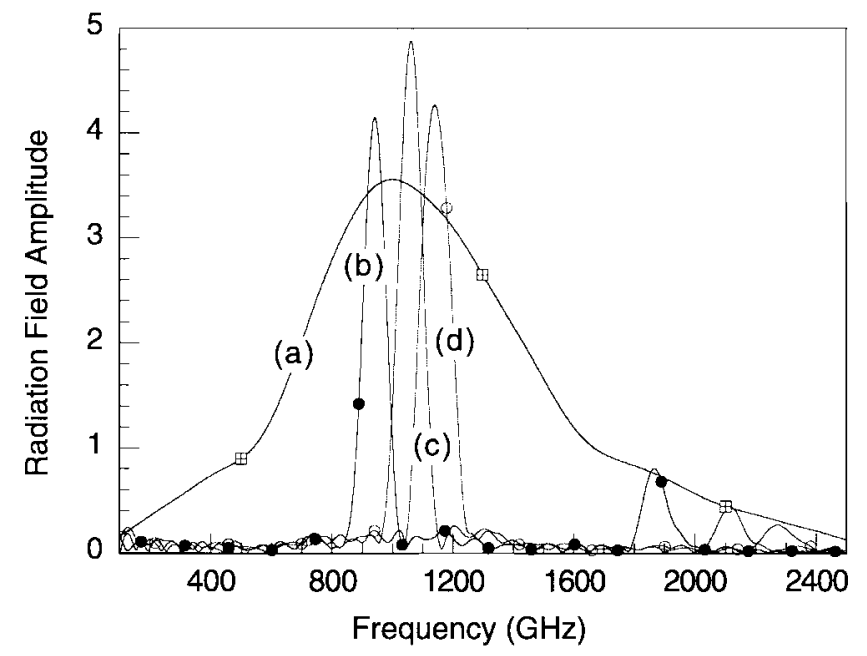

Fig. 8. FFT field spectral amplitude showing the tuning of narrow-band frequences through the changes of the spatial period: (a) Single-pulse, (b) $\Delta x=0.875 \mathrm{~mm}$, (c) $\Delta x=0.717 \mathrm{~mm}$, (d) $\Delta x=0.625 \mathrm{~mm}$.

compared to single-pulse radiation envelope. Section VI will explain this phenomena [37]. This figure clearly indicates the frequency tuning of the center wavelength of the narrow-band radiation by the spatial periodicity of the phase mask.

Fig. 9 shows the center frequency of the narrow-band radiation as a function of spatial period $\Delta x$. We have demonstrated a tuning range from $750 \mathrm{GHz}$ up to $1.3 \mathrm{THz}$ with our current mask and our pulse shaper design. As indicated by the fitted dashed line, the center frequency of the radiation field linearly depends on $\Delta x$. This is in reasonable agreement with (24) and our estimated pulse shaper parameters. We should point out that the tuning range of this technique is not limited to the range illustrated here. With proper design of the phase mask, we can expect an even wider frequency tuning range.

\section{OVERCOMing SATURATION OF TERAHERTZ RADIATION}

In this section, we are particularly interested in comparing the amplitude of terahertz radiation generated via singleand multiple-optical-pulse excitation. Similar to the previous

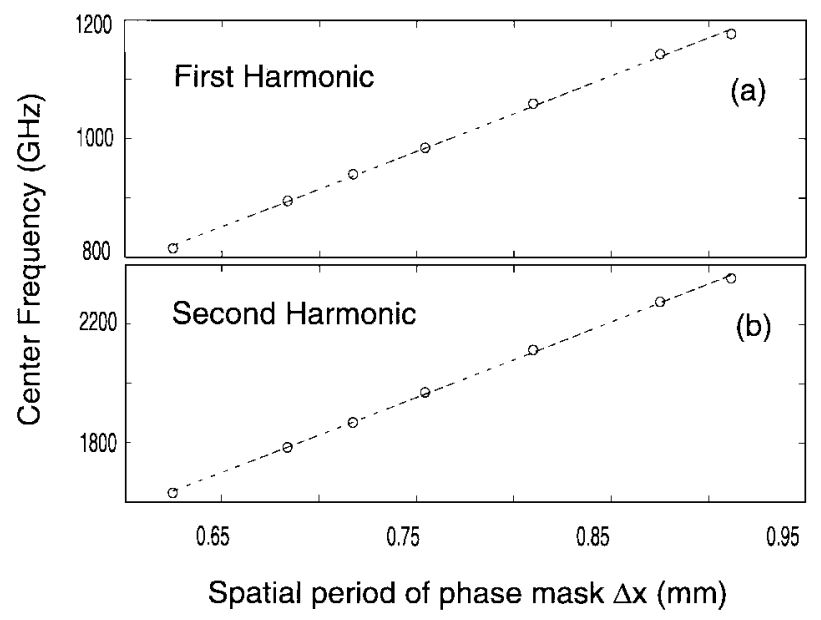

Fig. 9. Tuning range of the narrow-band terahertz radiation with current apparatus: (a) First-harmonic; (b) Second-harmonic.

section, we used a fixed phase mask to generate a multiple pulse sequence $\left(\nu_{0}=750 \mathrm{GHz}\right)$ [37].

As discussed and demonstrated in Section V, optical pulse shaping using a periodic phase filter should not change the amplitude of the narrow-band spectral peaks in $I(\omega)$ from the $I(\omega)$ curve corresponding to a single optical pulse of the same average power. This indicates that if the photoconducting antennas remain in the linear response regime, then the peak terahertz spectral amplitude at frequency $\nu_{0}$ excited by a multiple pulse sequence with repetition rate $\nu_{0}$ is the same as the terahertz spectral amplitude at frequency $\nu_{0}$ resulting from single-pulse excitation, provided that the overall optical fluence is unchanged. Thus, even though use of a multiple pulse sequence diminishes the peak optical intensity and peak terahertz field as viewed in the time domain, the multiple pulse character of the resultant waveforms lead to the same terahertz spectral amplitude at the selected frequency. This is indeed the case in our experiments at low optical powers $(P<12 \mathrm{~mW})$ as shown in Fig. 10(a), which plots the Fourier transformed terahertz power spectra under both single-pulse and multiplepulse excitations at an average optical power of $\sim 11 \mathrm{~mW}$. However, for higher optical power, a dramatic difference between single-pulse and multiple-pulse excitation is observed. Fig. 10(b) shows the FFT power spectra for a higher optical power excitation case of $\sim 44 \mathrm{~mW}$. The peak terahertz power spectral density resulting from multiple pulse excitation is increased by more than a factor of three compared to the case of single-pulse excitation. This surprising behavior, manifested in the enhancement of narrow-band terahertz generation by using multiple pulse optical excitation, arises due to avoidance of saturation mechanisms which limit the terahertz amplitude in the case of high-power single optical pulse excitation.

Fig. 11 shows a plot representing the terahertz spectral amplitude at $\nu_{0}=750 \mathrm{GHz}$ as a function of average optical power for both single- and multiple-pulse excitation. The solid symbols in the plot are derived directly from the FFT of the measured terahertz time-domain waveforms; the open symbols are obtained simply by recording the peak amplitudes of the detected time-domain terahertz waveforms versus power and then multiplying by the appropriate scale factors to convert 


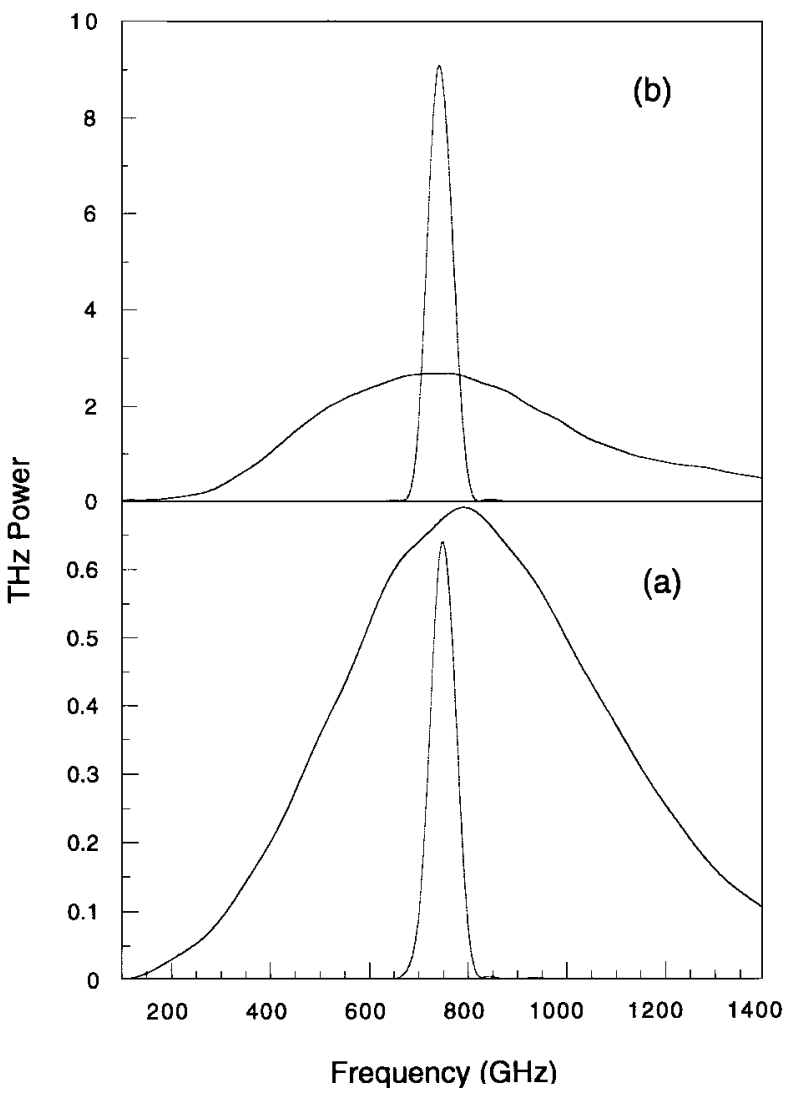

Fig. 10. Fourier transformed power spectrums of the terahertz radiations from broad-band single-pulse excitation and narrow-band multiple pulse excitation at an average power of (a) $P \sim 11 \mathrm{~mW}$, and (b) $P \sim 44 \mathrm{~mW}$ showing the enhancement of radiation by multiple pulse excitation at high powers.

from peak time-domain amplitude to spectral amplitude at $\nu_{0}$. Both single- and multiple-pulse plots show a linear dependence on optical power for average powers below $\sim 12 \mathrm{~mW}$. At higher powers, however, the terahertz amplitude for singlepulse excitation saturates, while the terahertz signal obtained using multiple pulse excitation remains linear with optical power. Note we took care to obtain data used in Figs. 10 and 11 in a single experimental session; the exact value of the saturation power could show some day-to-day variations due to variations in system alignment such as focusing onto the photoconductive antenna. We also chose the range of optical powers below the damage threshold to avoid antenna breakdown. The avoidance of saturation with multiple pulses arises because the peak optical intensity of the multiple pulse sequence is reduced nearly tenfold compared to the original single pulse, thus, scaling multiple pulse excitation into the linear response regime.

The scaling and saturation behavior of terahertz radiation with single optical pulse excitation has been studied both experimentally and theoretically. Saturation has been observed in a number of different transmitter structures [12], [20], [40]-[42]. In principle, the saturation at high optical power densities could result either from screening of the bias field due to the space-charge field or due to the radiation field itself. Saturation studies of dipole antennas and related structures have focused on space charge screening, which has led to

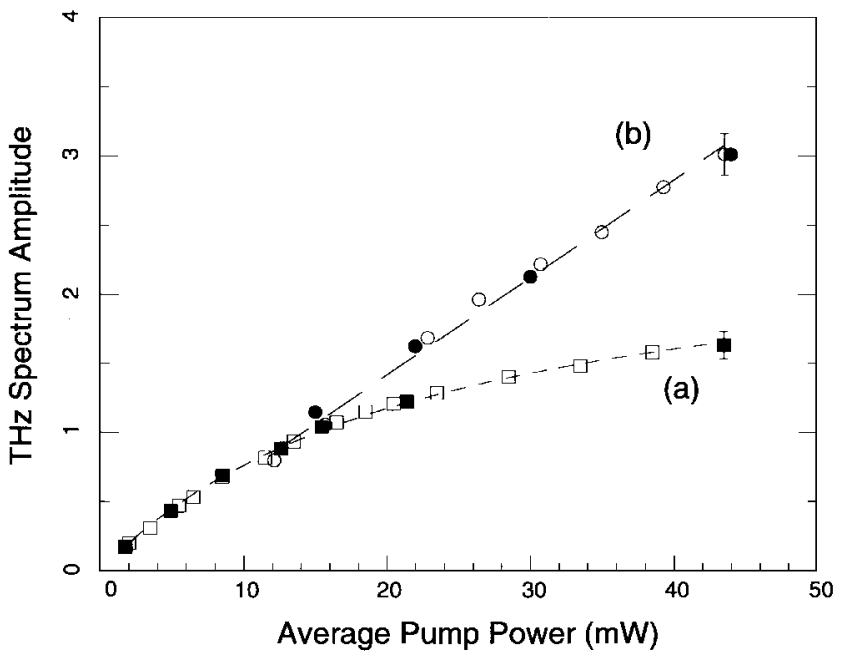

Fig. 11. Spectral amplitudes of the terahertz radiation as a function of the average excitation powers for the (a) single- and (b) multiple-optical pulse excitations. Solid symbols represent peak spectral amplitudes obtained directly from FFT. Oen symbols represent peak amplitudes in the time domain scaled to the spectral mplitudes at $\nu_{0}$ by multiplying by the appropriate scale factors. Dashed lies are fits to (25) for (a) using $F_{s} \sim 350 \mu \mathrm{J} / \mathrm{cm}^{2}$; linear fit for (b). Also shown in the figure are typical error bars.

significant saturation on an ultrafast time scale at carrier densities in the $10^{17}-10^{18} \mathrm{~cm}^{-3}$ range [41], [42]. In our highest power $(44 \mathrm{~mW})$ single-pulse experiments, the estimated carrier density is $\sim 3 \times 10^{18} \mathrm{~cm}^{-3}$. This suggests that space charge screening may also play a role in our single-pulse experiments, even though the magnitude of these effects will depend on the precise device geometry. With multiple pulse excitation each individual pulse generates substantially less electronhole pairs. Provided that the space-charge relaxation time is faster than the pulse repetition period as is likely given the subpicosecond carrier lifetime of our LT-GaAs transmitter, this strongly reduces screening effects due to the space-charge field. In the case of large aperture antennas, attention has focused on screening of the bias field by the radiation field. The terahertz field expected when saturation is due to screening by the radiation field is given by the following formula, valid when the aperture is large compared to the longest terahertz wavelength [20], [40]

$$
E_{r} \sim-E_{b} \frac{F(t) / F_{s}}{1+F(t) / F_{s}} .
$$

Here, $E_{b}$ is the bias field, $F(t)$ is the optical fluence, and the saturation fluence $F_{s}$ is a constant. Experimental values of $F_{s}$ are on the order of several tens of $\mu \mathrm{J} / \mathrm{cm}^{2}$ for semiinsulating GaAs and InP, respectively [12], [20], [40]. Our experiments use fluences as high as $700 \mu \mathrm{J} / \mathrm{cm}^{2}$, well above the saturation fluences reported for large aperture antennas; and a fit to our single-pulse data using Eq. (25) with $F_{s}=$ $350 \mu \mathrm{J} / \mathrm{cm}^{2}$ shows good agreement with our experimental results (see Fig. 11, dashed line). Thus, it seems likely that screening by the radiation field may be an important effect in our experiments, even though the large aperture results do not directly apply to our setup, where the dipole size is much less than the average terahertz average wavelength. The degree to which the bias field is actually screened by the radiation 
field for dipole antenna structures will have to be clarified by future investigation. In the experiments using multiple pulse excitation, the saturation is linked to peak optical power (or fluence per individual pulse). By spreading the optical excitation into multiple pulses resulting in a reduced peak radiation field, the saturation regime can be greatly extended.

We briefly remark on the relationship between the current work and previous experiments [30] which used similar femtosecond pulse sequences for impulsive excitation of coherent optical phonons in molecular crystals. These previous experiments were limited by optical damage; the use of lower intensity pulse sequences allowed greater optical fluences resulting in enhanced phonon amplitudes compared to singlepulse excitation. In the current experiments, we intentionally stay below the damage threshold but still attain enhanced terahertz generation by avoiding saturation effects.

Our current work does not distinguish between space-charge field and radiation field screening mechanisms, although multiple pulse excitation appears capable of alleviating both mechanisms. It may be possible to distinguish between these potential saturation mechanisms by performing further experiments in which both the pulse sequences and the material parameters are varied. Furthermore, we note that although the use of dipole antennas has limited the absolute field amplitude achieved in our current work, our proof-of-principle results can be extended to enhance terahertz power spectral densities achieved with high power, large aperture antennas. On the other hand, enhancement of the narrow-band generation efficiency with multiple pulses has an obvious cost-namely, loss of speed and absolute field amplitude due to the extended duration of the pulse trains. Thus, this technique will have greatest implications for applications requiring high-power, narrow-band terahertz radiation.

\section{CONCLUSION}

We have demonstrated experimentally that optical pulse shaping can be used to synthesize terahertz radiation waveforms generated from photoconductive antennas. By using femtosecond pulse sequences with controlled intensity modulations and timing, we have produced several examples of terahertz waveforms with desired amplitude and phase modulations. We have also presented a theory that relates the shaped terahertz waveform to the single-pulse terahertz system response and the cross correlation data for shaped and unshaped optical pulses. The theoretical predictions are in excellent agreement with the experimental results using no adjustable parameters. We show that one advantage of terahertz waveform shaping is the enhancement of narrow-band terahertz radiation generated by using optical pulse sequences through avoidance of saturation effects. This technique could have important implications for high-power terahertz applications such as terahertz nonlinear optics or particle accelerators. Our work can also be extended to allow generation of specially shaped mid-infrared radiation through difference frequency mixing of shaped optical pulses in second-order nonlinear crystals. In this case, the output radiation could be sensitive to the phase as well as the intensity and timing of the input optical waveforms, allowing even broader control of the output pulse profiles.

\section{ACKNOWLEDGMENT}

The authors would like to acknowledge helpful discussions with D. Grischkowsky and technical assistance from D. E. Leaird for fabricating phase masks.

\section{REFERENCES}

[1] G. Mourou, C. V. Stancampiano, A. Antonetti, and A. Orszag, "Picosecond microwave pulses generated with a subpicosecond laser driven semiconductor switch,” Appl. Phys. Lett., vol. 39, pp. 295-297, 1981.

[2] P. R. Smith, D. H. Auston, and M. C. Nuss, "Subpicosecond photoconducting dipole antennas," IEEE J. Quantum Electron., vol. 24, pp. 255-260, 1988.

[3] M. van Exter and D. R. Grischkowsky, "Characterization of an optoelectronic terahertz beam system," IEEE Trans. Microwave Theory Tech., vol. 38, pp. 1684-1691, 1990.

[4] D. Grischkowsky, "Nonlinear generation of subpicosecond pulses of $\mathrm{THz}$ electromagnetic radiation by optoelectronics-Application to timedomain spectroscopy," in Frontiers in Nonlinear Optics, H. Walther, N. Koroteev, and M. O. Scully, Eds. Philadelphia, PA: Inst. of Physics, 1993, pp. 196-228.

[5] D. Grischkowsky, S. Keiding, M. van Exter, and Ch. Fattinger, "Farinfrared time-domain spectroscopy with terahertz beams of dielectrics and semiconductors," J. Opt. Soc. Amer. B, vol. 7, pp. 2006-2014, 1990.

[6] J. F. Whitaker, F. Gao, and Y. Liu, "Terahertz bandwidth pulses for coherent time-domain spectroscopy," in Nonlinear Optics for HighSpeed Electronics and Optical Frequency Conversion, N. Peygambarian, H. Everitt, R. C. Eckardt, and D. D. Lowenthal, Eds. Bellingham, WA: SPIE, vol. 2145, 1994, pp. 168-177.

[7] J. A. Valdmanis, "Electro-optic measurement techniques for picosecond materials, devices and integrated circuits," in Semiconductors and Semimetals. New York: Academic, 1989, vol. 26, pp. 135-218.

[8] B. B. Hu and M. C. Nuss, "Imaging with terahertz waves," Opt. Lett. vol. 20, pp. 1716-1718, 1995.

[9] R. A. Cheville and D. Grischkowsky, "Time domain terahertz impulse ranging studies," Appl. Phys. Lett., vol. 67, pp. 1960-1962, 1995.

[10] X.-C. Zhang, B. B. Hu, J. T. Darrow, and D. H. Auston, "Generation of femtosecond electromagnetic pulses from semiconductor surfaces," Appl. Phys. Lett., vol. 56, pp. 1011-1013, 1990.

[11] J. T. Darrow, B. B. Hu, X.-C. Zhang, and D. H. Auston, "Subpicosecond electromagnetic pulses from large-aperture photoconducting antennas," Opt. Lett., vol. 16, pp. 323-325, 1990.

[12] B. I. Green, P. N. Saeta, D. R. Dykaar, S. Schmitt-Rink, and S. L. Chuang, "Far-infrared light generation at semiconductor surfaces and its spectroscopic applications," IEEE J. Quantum Electron., vol. 28, pp. 2302-2312, 1992.

[13] L. Xu, X.-C. Zhang, and D. H. Auston, "Terahertz beam generation by femtosecond optical pulses in electro-optic materials," Appl. Phys. Lett., vol. 61, pp. 1784-1786, 1992.

[14] Q. Wu and X.-C. Zhang, "Ultrafast electro-optic field sensors," Appl. Phys. Lett., vol. 68, pp. 1604-1606, 1996.

[15] M. C. Nuss, P. C. M. Planken, I. Brener, H. G. Roskos, M. S. C. Luo, and S. L. Chuang, "Terahertz electromagnetic radiation from quantum wells," Appl. Phys. B, vol. 58, pp. 249-259, 1994.

[16] C. Waschke, H. G. Roskos, R. Schwedler, K. Leo, H. Kurz, and K. Kohler, "Coherent submillimeter-wave emission from Bloch oscillations in a semiconductor superlattice," Phys. Rev. Lett., vol. 70, pp. 3319-3322, 1993.

[17] A. Bonvalet, M. Joffre, J. L. Martin, and A. Migus, "Generation of ultrabroadband femtosecond pulses in the mid-infrared by optical rectification of $15 \mathrm{fs}$ light pulses at $100 \mathrm{MHz}$ repetition rate," Appl. Phys. Lett., vol. 67, pp. 2907-2909, 1995.

[18] B. I. Green, J. F. Federici, D. R. Dykaar, R. R. Jones, and P. H Bucksbaum, "Interferometric characterization of 160 fs far-infrared light pulses," Appl. Phys. Lett., vol. 59, pp. 893-895, 1991.

[19] D. You, R. R. Jones, P. H. Bucksbaum, and D. R. Dykaar, "Generation of high-power sub-single-cycle 500-fs electromagnetic pulses," Opt. Lett., vol. 18, pp. 290-292, 1993.

[20] J. T. Darrow, X.-C. Zhang, D. H. Auston, and J. D. Morse, "Saturation properties of large-aperture photoconducting antennas," IEEE J. Quantum Electron., vol. 28, pp. 1607-1616, 1992. 
[21] A. S. Weling, B. B. Hu, N. M. Froberg, and D. H. Auston, "Generation of tunable narrow-band $\mathrm{THz}$ radiation from large aperture photoconducting antennas," Appl. Phys. Lett., vol. 64, pp. 137-139, 1994.

[22] N. M. Froberg, B. B. Hu, X.-C. Zhang, and D. H. Auston, "Timedivision multiplexing by a photoconducting antenna array," Appl. Phys. Lett., vol. 59, pp. 3207-3209, 1991.

[23] X.-C. Zhang and D. H. Auston, "Generation of steerable submillimeter waves from semiconductor surfaces by spatial light modulators," Appl. Phys. Lett., vol. 59, pp. 768-770, 1991.

[24] P. C. M. Planken, I. Brenar, M. C. Nuss, M. S. C. Luo, and S. L. Chuang, "Coherent control of terahertz charge oscillations in a coupled quantum well using phase-locked optical pulses," Phys. Rev. B, vol. 48, pp. 4903-4906, 1993.

[25] I. Brener, P. C. Planken, M. C. Nuss, L. Pfeiffer, D. E. Leaird, and A. M. Weiner, "Repetitive excitation of charge oscillations in semiconductor heterostructures," Appl. Phys. Lett., vol. 63, pp. 2213-2215, 1993.

[26] I. Brener, P. C. M. Planken, M. C. Nuss, M. S. C. Luo, S. L. Chuang, L. Pfeiffer, D. E. Leaird, and A. M. Weiner, "Coherent control of terahertz emission and carrier populations in semiconductor heterostructures," $J$. Opt. Soc. Am. B, vol. 11, pp. 2457-2469, 1994

[27] A. M. Weiner, J. P. Heritage, and E. M. Kirschner, "High-resolution femtosecond pulse shaping,” J. Opt. Soc. Amer. B, vol. 5, pp. 1563-1572, 1988.

[28] A. M. Weiner, D. E. Leaird, J. S. Patel, and J. R. Wullert, "Programmable shaping of femtosecond optical pulses by use of a 128-element liquid crystal phase modulator," IEEE J. Quantum Electron., vol. 28, pp. 908-920, 1992

[29] M. M. Wefers and K. A. Nelson, "Generation of high-fidelity programmable ultrafast optical waveforms," Opt. Lett., vol. 20, pp. $1047-1049,1995$

[30] A. M. Weiner, D. E. Leaird, G. P. Wiederrecht, and K. A. Nelson, "Femtosecond pulse sequences used for optical manipulation of molecular motion," Science, vol. 247, pp. 1317-1319, 1990

[31] A. M. Weiner, "Femtosecond optical pulse shaping and processing," Progress in Quantum Electron., vol. 19, pp. 161-238, 1995.

[32] A. M. Weiner and D. E. Leaird, "Generation of terahertz-rate trains of femtosecond pulses by phase only filtering," Opt. Lett., vol. 51, pp. $51-53,1990$

[33] A. M. Weiner, D. E. Leaird, G. P. Wiederrecht, and K. A. Nelson, "Femtosecond multiple-pulse impulsive stimulated Raman scattering spectroscopy," J. Opt. Soc. Amer., vol. 8, pp. 1264-1275, 1991.

[34] A. M. Weiner, S. Oudin, D. E. Leaird, and D. H. Reitze "Shaping of femtosecond pulses using phase-only filters designed by simulated annealing," J. Opt. Soc. Amer. A, vol. 10, pp. 1112-1120, 1993.

[35] K. Nelson, "Coherent control: Optics, molecules, and materials," Ultrafast Phenomena IX, G. A. Mourou et al., Eds. Berlin, Germany: Springer-Verlag, 1994, pp. 47-49.

[36] S. H. Shi and H. Rabitz, "Optimal control of selective vibrational excitation of harmonic molecules: Analytic solution and restricted forms for the optimal fields," J. Chem. Phys., vol. 92, pp. 2927-2937, 1990.

[37] Y. Liu, S.-G. Park, and A. M. Weiner, "Enhancement of narrowband terahertz radiation from photoconducting antennas by optical pulse shaping," Opt. Lett., vol. 111, p. 1762, 1996.

[38] B. B. Hu, J. T. Darrow, X.-C. Zhang, and D. H. Auston, "Optically steerable photoconducting antennas," Appl. Phys. Lett., vol. 56, pp. 886-888, 1990.

[39] S. Gupta, M. Frankel, J. A. Valdmanis, J. F. Whitaker, G. Mourou, F. W. Smith, and A. R. Calawa, "Subpicosecond carrier lifetime in GaAs grown by molecular beam epitaxy at low temperatures," Appl. Phys. Lett., vol. 59, pp. 3276-3278, 1991.

[40] P. K. Benicewicz, J. P. Roberts, and A. J. Taylor, "Scaling of terahertz radiation from large-aperture biased photoconductors," J. Opt. Soc. Amer. B, vol. 11, pp. 2533-2546, 1994.

[41] J. E. Pedersen, V. G. Lyssenko, J. M. Hvam, P. H. Jepsen, S. R. Keiding, C. B. Sorensen, and P. E. Lindelof, "Ultrafast local field dynamics in photoconductive THz antennas," Appl. Phys. Lett., vol. 62, pp. 1265-1267, 1993.

[42] W. Sha, J. Rhee, T. Norris, and W. J. Schaff, "Transient carrier and field dynamics in GaAs quantum well parallel transport: From the ballistic to the quasiequilibrium regime," IEEE J. Quantum Electron., vol. 28, pp. 2445-2455, 1992.
Yongqian Liu was born in Xian, China, in 1964. He graduated from the University of Science and Technology of China in 1987 with the B.S. degree in physics. From 1987 to 1990, he worked at the Institute of Physics, Chinese Academy of Sciences. He received the M.S. in EECS degree and the Ph.D. degree in physics from the University of Michigan, Ann Arbor, in 1995.

He then spent his time at the School of Electrical and Computer Engineering, Purdue University working as a Post-Doctoral research associate. His current research interests cover ultrafast optics, optoelectronic devices, terahertz optical systems and its applications, optical and terahertz pulse processing, coherent control of charge oscillations in semiconductor quantum devices, fiber and mm-submm-wave communications.

Dr. Liu was a Pre-Doctoral Fellow at the University of Michigan, winner of the the Kent M. Terwilliger Most Outstanding Ph.D. Thesis Award and Thesis Grant from the Rackham Graduate School, the University of Michigan.

Sang-Gyu Park was born in Seoul, Korea, in 1967. He received the B.S. and M.S. degrees in electrical engineering from Seoul National University in 1990 and 1992, respectively, and is currently pursuing the Ph.D degree in electrical engineering at Purdue University, West Lafayette, IN.

His research interests include ultrafast optics and semiconductor physics.

A. M. Weiner was born in Boston, MA, in 1958. He received the Sc.D. degree in electrical engineering from M.I.T., Cambridge, MA, in 1984.

From 1979 through 1984, he was a Fannie and John Hertz Foundation Graduate Fellow at M.I.T. His doctoral thesis, for which he was awarded the 1984 Hertz Foundation Doctoral Thesis Prize, dealt with femtosecond pulse compression (including generation of the shortest optical pulses reported up to that time) and measurement of femtosecond dephasing in condensed matter. In 1984, he joined Bellcore where he conducted research on ultrafast optics, including shaping of ultrashort pulses, nonlinear optics and switching in fibers, and spectral holography. In 1989, he became Manager of the Ultrafast Optics and Optical Signal Processing Research District. He assumed his current position as Professor of Electrical and Computer Engineering at Purdue University in October 1992. Since August 1996, he has also been serving as Director of Graduate Admissions for the School of Electrical and Computer Engineering, His current research interests center on holography of ultrashort pulses, high-speed optical communications, and applications of pulse shaping to femtosecond spectroscopy and nonlinear optics.

Prof. Weiner is currently Topical Editor of Optics Letters and previously served as Associate Editor of IEEE Journal OF QuANTUM EleCtronics and of IEEE Photonics TeChNology LetTers. He has also served as vicechairman of the Gordon Conference on Nonlinear Optics and Lasers, chair of the Ultrafast Phenomena Technical Group of the Optical Society of America (OSA), chair of the OSA Lomb Medal Committee, member of the IEEE Education Medal Committee and of the IEEE Quantum Electronics Award Committee, and member of numerous conference committees. He was Program Co-Chair for the 1996 Conference on Laser and Electro-Optics. In 1988-1989, he served as IEEE Lasers and Electro-Optics Society Traveling Lecturer, and in 1990 he was awarded the Adolph Lomb Medal of the OSA for noteworthy contributions to optics made before reaching the age of 30 . Dr. Weiner has authored approximately 160 conference and university talks and approximately 80 technical articles, including four book chapters, and is holder of five U.S. patents. He is a Fellow of the OSA and the IEEE LEOS Board of Governors. 